\title{
GLP-1 analogue CJC-1131 prevents amyloid $\beta$ protein-induced impirments of spatial memory and synaptic plasticity in rats
}

\author{
Sheng-Xiao Zhang ${ }^{\mathrm{a}, 1}$, Hong-Yan Cai ${ }^{\mathrm{b}, 1}$, Xiao-Wen $\mathrm{Ma}^{\mathrm{a}}$, Li Yuan ${ }^{\mathrm{c}}$, Jun Zhang ${ }^{\mathrm{c}}$, \\ Zhao-Jun Wang ${ }^{\mathrm{c}}$, Yu-Feng $\mathrm{Li}^{\mathrm{a}}$, Jin-Shun $\mathrm{Qi}^{\mathrm{c}}{ }^{\mathrm{c}}$, \\ a Department of Rheumatology, The Second Hospital of Shanxi Medical University, 56 Xinjian South Road, Taiyuan, Shanxi 030001, China \\ ${ }^{\mathrm{b}}$ Department of Microbiology and Immunology, Shanxi Medical University, 56 Xinjian South Road, Taiyuan, Shanxi 030001, China \\ ${ }^{\mathrm{c}}$ Department of Physiology and National Key Discipline of Physiology, Shanxi Medical University, 56 Xinjian South Road, Taiyuan, Shanxi 030001, China
}

\section{A R T I C L E I N F O}

\section{Article history:}

Received 21 November 2016

Received in revised form 4 March 2017

Accepted 8 March 2017

Available online 15 March 2017

\section{Keywords:}

CJC-1131

Amyloid $\beta$ protein

Spatial memory

Morris water maze

Long-term potentiation

Synaptic plasticity

\begin{abstract}
A B S T R A C T
Although amyloid $\beta$ protein $(A \beta)$ has been recognized as one of the main pathological characteristics in the brain of Alzheimer's disease (AD), the effective strategies against $A \beta$ neurotoxicity are still deficient up to now. Glucagon-like peptide 1 (GLP-1), a natural gut hormone, was found to be effective in modulating insulin signaling and neural protection, but short half-life limited its clinical application in AD treatment. CJC-1131, a newly designed GLP-1 analogue with very longer half-life, has shown good effectiveness in the treatment of type 2 diabetes mellitus (T2DM). However, it is unclear whether CJC-1131 could alleviate $A \beta$-induced neurotoxicity in cognitive behavior and electrophysiological property. The present study investigated the effects of $C J C$ - 1131 on the $A \beta$-induced impairments in spatial memory and synaptic plasticity of rats by using Morris water maze test and in vivo field potential recording. The results showed that $A \beta 1-42$-induced increase in the escape latency of rats in hidden platform test and decrease in swimming time percent in target quadrant were effectively reversed by CJC-1131 pretreatment. Further, CJC-1131 prevented against $A \beta 1-42$-induced suppression of hippocampal long term potentiation (LTP). In addition, $A \beta 1-42$ injection resulted in a significant decrease of $p$-PKA in the hippocampus, which was effectively prevented by CJC-1131 treatment. These results indicated that CJC- 1131 protected the cognitive function and synaptic plasticity of rats against $A \beta$-induced impairments, suggesting that GLP1 analogue $\mathrm{CJC}-1131$ might be potentially beneficial to the prevention and treatment of AD, especially those with T2DM or blood glucose abnormality.
\end{abstract}

(C) 2017 Elsevier B.V. All rights reserved.

\section{Introduction}

Alzheimer's disease (AD), the leading cause of the senile dementia in the world, is a chronic, incurable neurodegenerative disease, with gradually decayed cognitive function and impaired learning and memory. One of the main pathological characteristics of AD is the accumulation of senile plaques in the brain with $A D$. Senile plaques are mainly composed of amyloid beta protein $(A \beta)$, primarily responsible for the neuronal death $[1,2]$. It has been universally reported that not only natural amyloid beta proteins such as $A \beta 1$ 42 [3], $A \beta 1-40$ [4] but also synthetic amyloid beta proteins like

\footnotetext{
* Corresponding author at: Department of Physiology and Neurobiology Key Laboratory of Cellular Physiology, Ministry of Education, Shanxi Medical University, Taiyuan, Shanxi, 030001, China.

E-mail address: jinshunqi2009@163.com (J.-S. Qi).

1 With the same contribution.
}

http://dx.doi.org/10.1016/j.bbr.2017.03.018

0166-4328/@ 2017 Elsevier B.V. All rights reserved.
$A \beta 25-35$ [5] and even $A \beta 31-35$ [6] can injure neurons in vivo and in vitro. Unluckily, there is no effective strategy to antagonist the neurotoxicity of $A \beta$ up to now.

It is interesting that $\mathrm{AD}$ is closely related to type 2 diabetes mellitus (T2DM) in many ways. For example, the prevalence of both diseases (AD and T2DM) was increased exponentially with aging; T2DM raised 1.5-2.5-fold risk of dementia [7,8], while AD patients often have blood glucose abnormalities. The pathological characteristics of both diseases also have much in common. The loss and dysfunction of $\beta$-cell in T2DM is related to amyloid deposition [9], which is pretty similar to the aggregated $A \beta$ in the AD brain. Not only $A \beta$, but also hyperphosphorylated tau protein appeared in both AD and T2DM [10]. Moreover, streptozotocin (STZ), used to damage $\beta$ cells of the pancrea and produce diabetes, also impaired cognition and led to AD model [11]. Schwartz [12] found that the decrease of glucose utilization and the impairment of energy metabolism in AD patients might be related to the damage of the insulin signaling pathway. All of these facts indicate a close 
association between AD and T2DM. Therefore, AD has been considered or named as type 3 diabetes [13]. Importantly, some insulin signal normalizing gut hormones used to treat T2DM such as glucagon-like peptide 1 (GLP-1) have been found to be effective in preventing AD progress. However, natural GLP-1 has a very short half-life of about $2 \mathrm{~min}$ [14], due to the rapid degradation by enzyme dipeptidyl peptidase IV (DPP IV), which seriously limits the application of GLP-1 in the clinic.

CJC-1131, a new chemical-modified GLP-1 analogue containing a D-alanine substitution and a maleimidopropylamido attachment to albumin [15], was found to be effective in resisting DPP IV degradation and has a very longer half-life up to 221-353 h [16]. Except for a high dose-related gastrointestinal complaint, no obvious side effect of CJC-1131 was observed in clinical application [17]. The benefits of CJC-1131 in T2DM treatment have been praised, which significantly reduced HbA1c and fasting blood glucose level, improved glucose tolerance and reduced body weight without hypoglycemia adverse effects $[17,18]$. However, the neuroprotective effects of CJC-1131, as a new GLP-1 analogue with longer half-life, have not been examined up to now. Therefore, the present study explored the neuroprotective effects of intra-hippocampal $\mathrm{CJC}-1131$ injection against $\mathrm{A} \beta 1$-42-induced impairments in spatial learning and memory of rats. Furthermore, we recorded in vivo hippocampal long-term potentiation (LTP) in the CA1 region of rats to clarify possible association between behavioral changes and electrophysiological properties. Finally, the level of p-PKA in the hippocampus was examined to illuminate the possible molecular mechanism of CJC-1131 in neuroprotection.

\section{Materials and methods}

\subsection{Animals and drugs}

Forty adult male Sprague-Dawley (SD) rats (180-220 g) were provided by the Animal Research Center of Shanxi Medical University. All animal procedures were carried out according to the guidelines of Shanxi Committee on Ethics of Animal Research. The rats were kept in a room with $12 \mathrm{~h}$ light and $12 \mathrm{~h}$ dark cycle at the temperature of $25 \pm 2{ }^{\circ} \mathrm{C}$ and humidity of $60 \%-80 \%$. A $\beta 1-42$ was ordered from Sigma (Sigma-Aldrich, Germany) and CJC-1131 was synthesized by Sangon (Sangon-Biotech, Shanghai, China). Both of them were dissolved in saline $(2.5 \mathrm{nmol} / \mu \mathrm{l})$ and stored at $-20^{\circ} \mathrm{C}$.

\subsection{Surgery and intra-hippocampal injection}

All rats were randomly divided into four groups: vehicle + saline, $A \beta 1-42+$ saline, vehicle $+C J C-1131$, and $A \beta 1-42+C J C-1131$. To minimize suffering, all surgery was performed under the anesthesia by chloral hydrate intra-peritoneal injection $(0.3 \mathrm{~g} / \mathrm{kg})$. The SD rat was placed in a stereotaxic apparatus (Narishige, Tokyo, Japan). Drugs were administered into the bilateral hippocampi at the following coordinates [19-21]: anterior/posterior: $-3.0 \mathrm{~mm}$, lateral/medial: $\pm 2.2 \mathrm{~mm}$, dorsal/ventral: $-3.0 \mathrm{~mm}$, with an injection rate of $0.2 \mu \mathrm{l} / \mathrm{min}$ under the control of a micro pump (RWD Life Science Ltd., Shenzhen, China). CJC-1131 ( $2 \mu \mathrm{l})$ or saline $(2 \mu \mathrm{l})$ was firstly injected into the bilateral hippocampi. 15 min later, $A \beta 1$ $42(2 \mu \mathrm{l})$ or vehicle $(2 \mu \mathrm{l})$ was applied. To make sure that drugs were fully dispersed into the hippocampus, a 5 min-retention of the injection syringe in the brain was given after every injection.

\subsection{Morris water maze test}

Morris water maze (MWM) test was performed two weeks after surgery $[20,22,23]$. The maze (Zhenghua Bio Instrument Ltd., China) was a stainless pool $(150 \mathrm{~cm}$ in diameter and $50 \mathrm{~cm}$ in height $)$ with tap water kept at $25 \pm 2^{\circ} \mathrm{C}$. An escape platform $(14 \mathrm{~cm}$ in diameter,
$29 \mathrm{~cm}$ in height) was located in the center of one quadrant and was below the surface of the water about $1 \mathrm{~cm}$. The movement of rat was monitored and recorded by camera above the maze and the signals were analyzed by behavioral analysis software (Noldus Information Technology, Ethovision 3.0, Wageningen, Netherlands). The escape latency and distance to find the escape platform, the percentages of time and distance in target quadrant, and the swimming speed were calculated. In the hidden platform test, rat was placed into the water facing the wall of the pool at a random quadrant. If it didn't find the hidden platform in $120 \mathrm{~s}$, the rat was led to the platform and stayed on the platform for $10 \mathrm{~s}$. Rats were trained four times per day for 5 days. In the probe trial on the sixth day, the platform was removed and the rat was allowed to swim in the pool freely for $120 \mathrm{~s}$. After that, visible platform test was performed, in which the platform was elevated above the water surface about $1 \mathrm{~cm}$.

\subsection{In vivo hippocampal LTP recording}

After the MWM test, SD rats were anesthetized (urethane, $1.5 \mathrm{~g} / \mathrm{kg}$ ) and placed in a stereotaxic apparatus. An electric blanket was used under body to maintain the rats' temperature at $37-38^{\circ} \mathrm{C}$. The skull of rat was drilled, and a bound stimulating/recording electrode was inserted into the Schaffer collateral/CA1 region. Continuous single stimuli with an interval of $30 \mathrm{~s}$ were applied to Schaffer to induce field excitatory postsynaptic potentials (fEPSPs) in the CA1 region. Basic fEPSPs were firstly recorded for $30 \mathrm{~min}$ as control. Two paired-pulse stimuli were given to trigger pairedpulse facilitation (PPF), and high-frequency stimulation (HFS) with three bursts were given to induce LTP. Each burst had 20 pulses. LTP was recorded for at least $1 \mathrm{~h}$ after HFS [19,24].

\subsection{Western blot assay}

After the electrophysiological experiment, the hippocampi of rats were instantly dissected and frozen at $-80^{\circ} \mathrm{C}$. Western blot was performed as previously described [19]. Protein concentration was detected with bicinchoninic acid protein assay kit. Total protein was separated by $12 \%$ SDS-PAGE and transferred to PVDF membrane. Nonspecific binding was blocked with 5\% BSA in Trisbuffered saline containing 0.05\% Tween-20 (TBST). Target proteins were detected by GAPDH anti-rat monoclonal antibody (dilution 1:1000; ZSGB-BIO, Beijing, China), phosphorylated PKA anti-rat monoclonal antibody (dilution 1:1000; Abcam, Cambridge, MA, USA) and PKA anti-rat monoclonal antibody (dilution 1:1000; Abcam, Cambridge, MA, USA). Secondary antibody of anti-rabbit immunoglobulin $G$ coupled to horseradish peroxidase (dilution 1:5000) was obtained from ZSGB-BIO. The membrane was rinsed with TBST and the immunocomplex was visualized by using an enhanced chemiluminescence detection kit (Beyotime, Inc). The signals of the membrane were scanned with the FluorChem Scanner and quantified with the Alpha View SA software.

\subsection{Data analysis}

All data were represented as the mean \pm SEM. For the escape latency in the hidden platform test, three-way repeated measures analysis of variance was used. Other data were analyzed by twoway repeated measures analysis of variance. The software for the statistics was SPSS 13.0 and SigmaPlot 12.3. $\mathrm{p}<0.05$ was taken as statistical significance. 

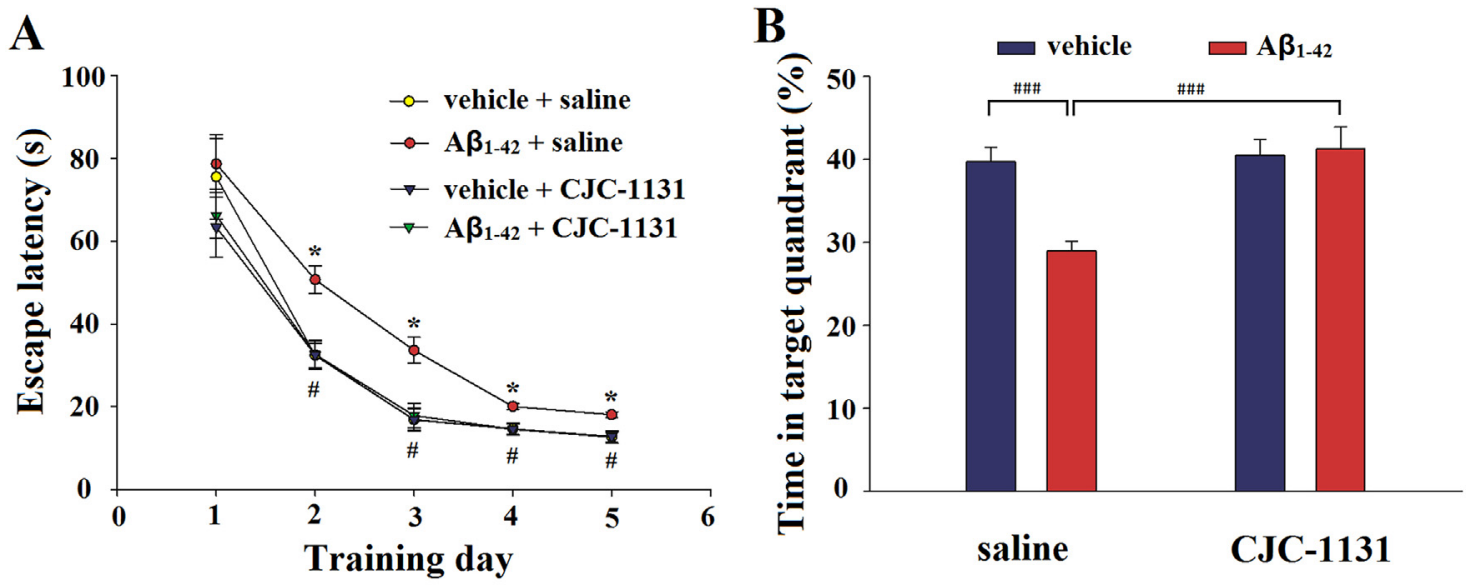

C

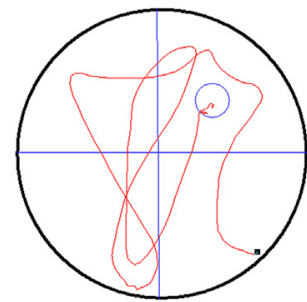

vehicle + saline

D

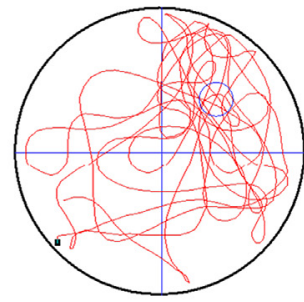

vehicle + saline

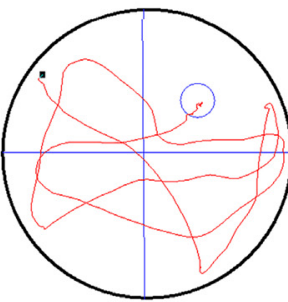

vehicle + CJC-1131

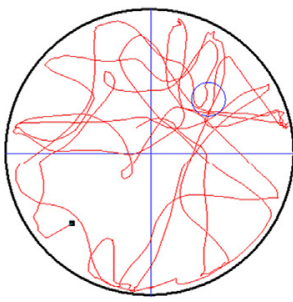

vehicle + CJC-1131

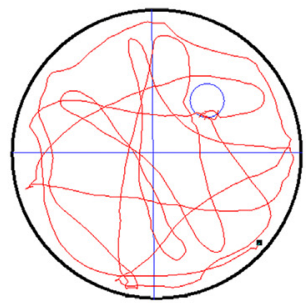

$A \beta_{1-42}+$ saline

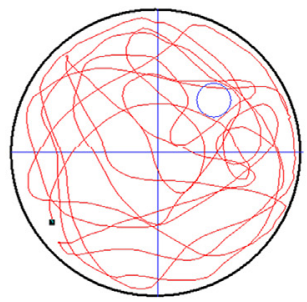

$A \beta_{1-42}+$ saline

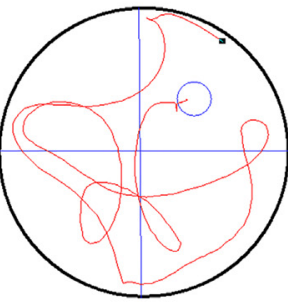

$A \beta_{1-42}+\mathbf{C J C}-1131$

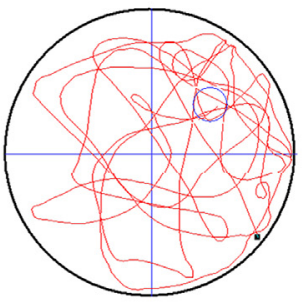

$A \beta_{1-42}+\mathbf{C J C}-1131$

Fig. 1. The effects of $A \beta 1-42$ and CJC-1131 on the spatial learning and memory of rats in Morris water maze tests.

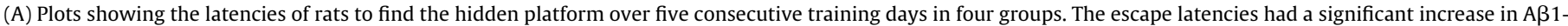

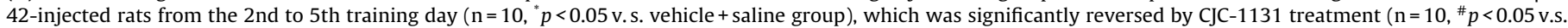

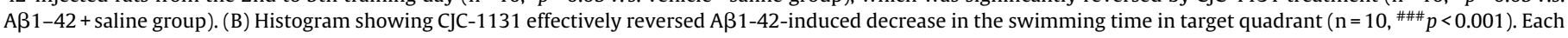

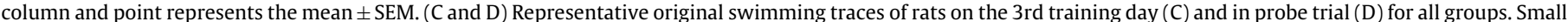
circles in 1st quadrant represent the water platform, and black dots represent the locations where the rats were placed.

\section{Results}

\subsection{CJC-1131 effectively prevented A $\beta 1-42$-induced impairments in spatial learning and memory of rats}

As shown in Fig. 1A, the escape latencies of rats to find the hidden platform decreased during 5 consecutive training days $\left(F_{(4,195)}=143.472 ; p<0.001\right)$. The main effect of $A \beta 1-42$ injection (A $\beta 1-42$ vs. vehicle) was significant on the escape latency $\left(\mathrm{F}_{(1,38)}=8.889 ; p<0.01\right)$. The main effect of $\mathrm{CJC}-1131$ treatment (CJC-1131 vs. saline) was also significant $\left(\mathrm{F}_{(1,38)}=15.313 ; p<0.001\right)$. The interaction between $A \beta 1-42$ injection and $C J C-1131$ treatment was significant $\left(\mathrm{F}_{(1,38)}=6.567 ; p<0.05\right)$. The mean escape latency of rats in $A \beta 1-42$ injection group was significantly longer than that in vehicle injection group $(p<0.05)$, showing that the rats with $A \beta 1-42$ intra-hippocampal injection learned more slowly than the rats by vehicle injection. CJC-1131 treatment had no significant effect on the escape latency of rats compared with saline treatment group $(p>0.05)$, but effectively decreased the escape latency of rats in $\mathrm{CJC}-1131+\mathrm{A} \beta 1-42$ group, suggesting that $\mathrm{CJC}-1131$ prevented against $A \beta 1-42$-induced deficit in learning ability.

In probe trials shown in Fig. $1 \mathrm{~B}$, two-way ANOVA showed that the percentage of time in the target quadrant (platform previously located) had significant main and interaction effects between $A \beta 1$ 42 and $C J C-1131$ groups $\left(A \beta 1-42\right.$ injection: $F_{(1,38)}=8.575 ; p<0.01$; CJC-1131 treatment: $\mathrm{F}_{(1,38)}=10.890 ; p<0.01 ; A \beta 1-42$ by CJC -1131 interaction: $\left.F_{(1,38)}=6.488 ; p<0.05\right)$. Tukey's post hoc test showed $A \beta 1-42$ injection resulted in a significant decrease in the percentage of time in the target quadrant $(p<0.001)$, which was effectively reversed by CJC-1131 treatment $(p<0.001)$.

To confirm whether CJC-1131 and A $31-42$ affected the motor ability of rats, the average swimming speeds of rats were compared. The results showed that there was no significant difference in swimming speeds among all groups $(p>0.05)$. In addition, the results of visible platform test indicated that neither CJC-1131 nor $A \beta 1-42$ affected the vision of rats, since the mean escape latency in the visible platform test was nearly the same, being approximately $12.99 \mathrm{~s}$ in all groups. 
A

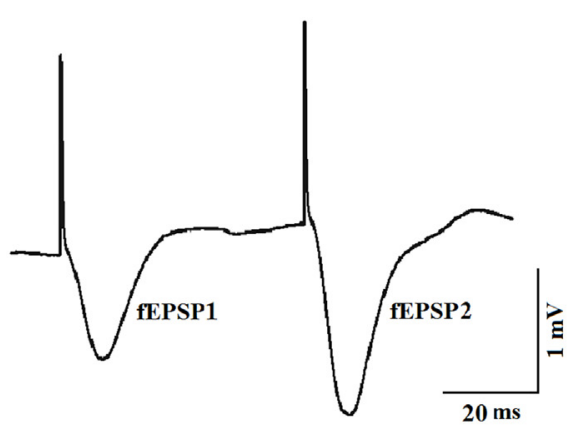

C

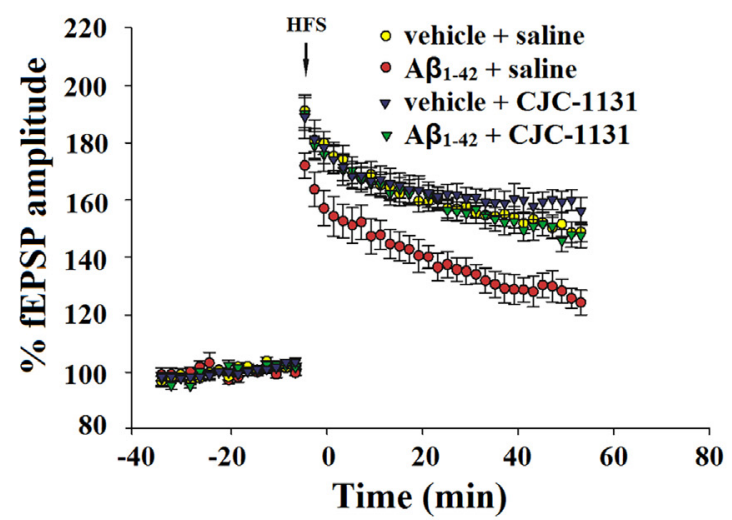

E

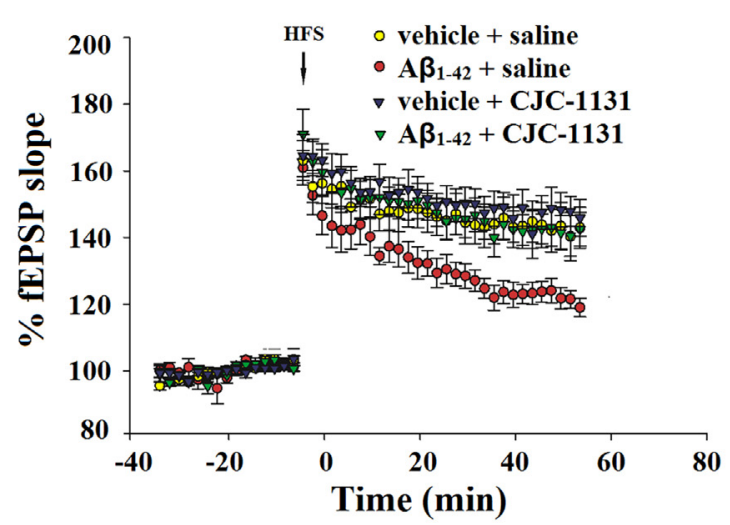

B

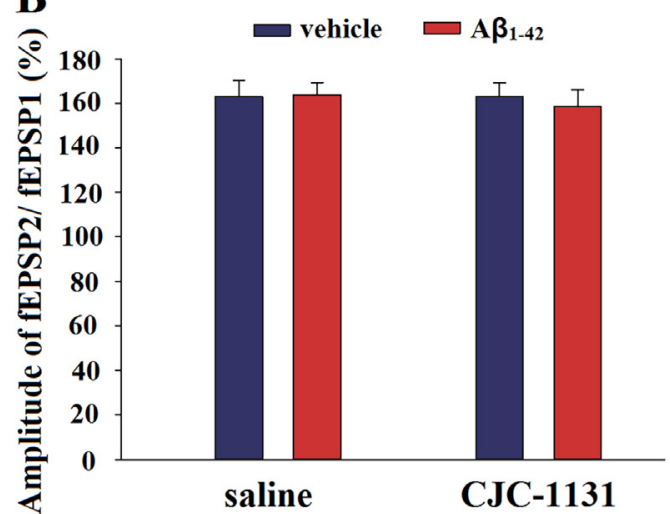

D

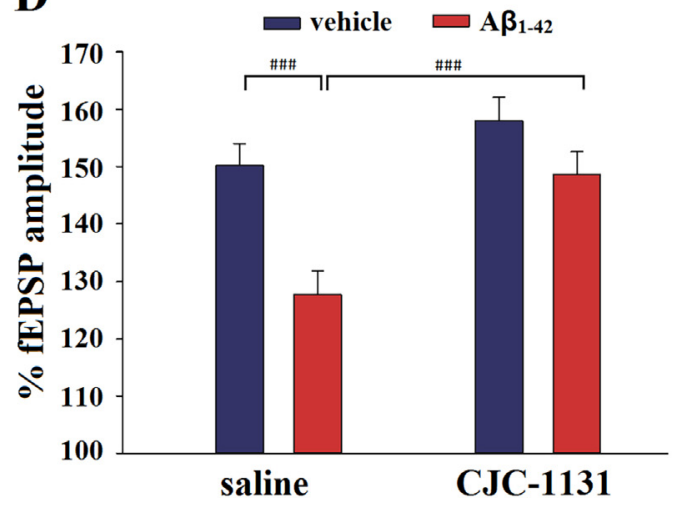

F

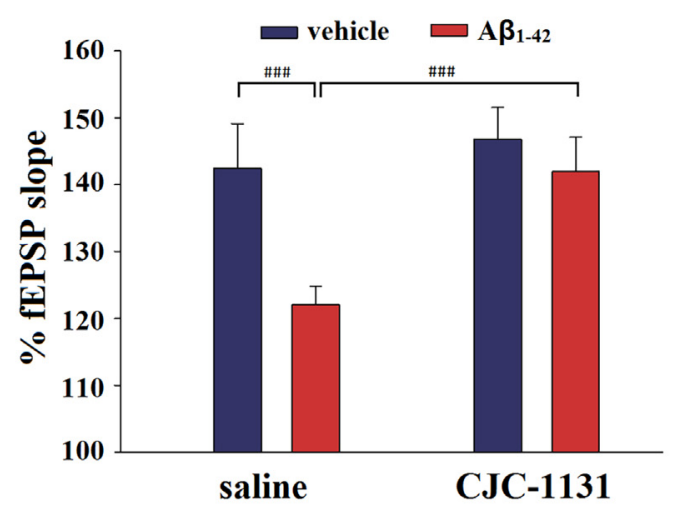

G

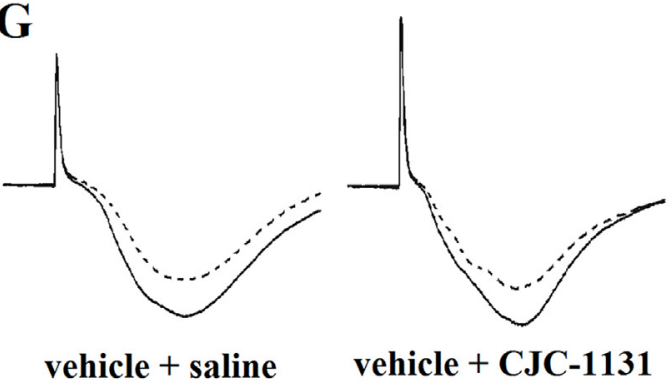

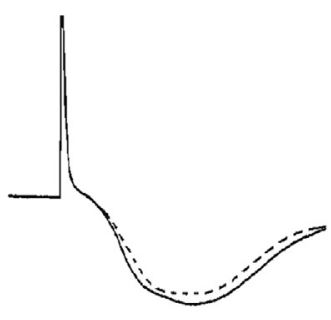

$\mathrm{A} \beta 1-42+$ saline

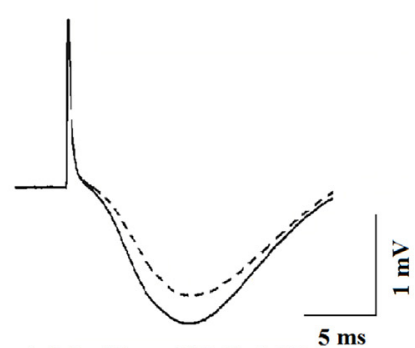

$\mathrm{A} \beta 1-42$ + CJC-1131

Fig. 2. The effects of $A \beta 1-42$ and CJC-1131 on in vivo hippocampal LTP in the CA1 region of rats.

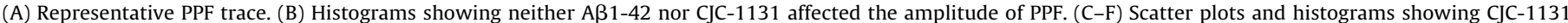

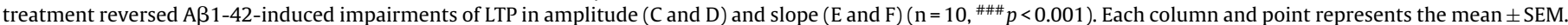

(G) Typical fEPSP traces recorded before (dash line) and $60 \mathrm{~min}$ after (solid line) HFS in each group. 
A

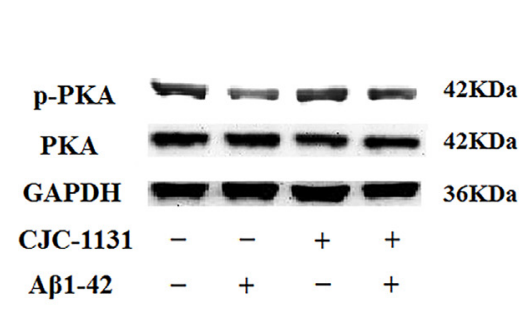

B

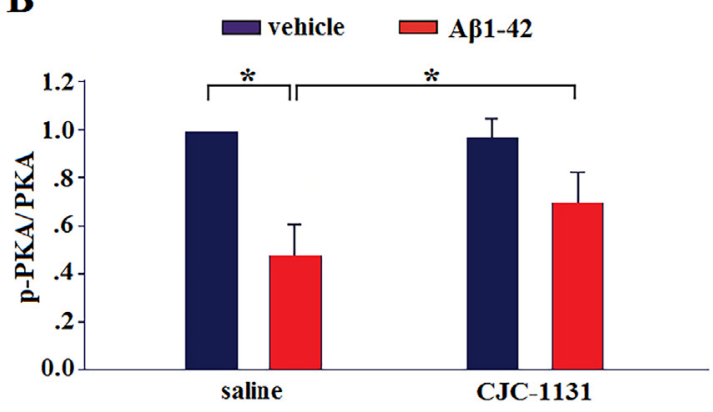

Fig. 3. CJC-1131 treatment restored the level of p-PKA in the hippocampus.

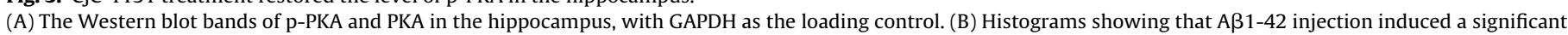
decrease in the level of p-PKA, which was reversed by CJC-1131 treatment $\left(n=6,{ }^{*} p<0.05\right)$. Each column and point represents the mean \pm SEM.

\subsection{CJC-1131 reversed $A \beta 1-42$-induced suppression of hippocampal LTP without affecting PPF}

In view of the close relationship between spatial cognition and hippocampal synaptic plasticity, we further examined the effects of A $1-42$ injection and CJC-1131 treatment on hippocampal synaptic plasticity. Paired-pulse facilitation (PPF) was first observed before HFS to clarify whether $A \beta 1-42$ and CJC-1131 could affect the presynaptic transmitter release in the hipocampal CA1 region. Fig. 2A shows a representative PPF trace, with always higher amplitude of fEPSP2 than fEPSP1. Two-way ANOVA showed that there was no main effect on the ratio of PPF in both $A \beta 1-42$ injection and CJC-1131 treatment (Fig. 2B), indicating that pre-synaptic neurotransmitter release was not changed by $A \beta 1-42$ and CJC-1131. Then, we compared HFS-induced LTP by measuring the amplitude (Fig. 2C, D) and slope (Fig. 2E, F) percentages of fEPSPs. Two-way ANOVA showed that the amplitude of post-HFS had significant main and interaction effects between $A \beta 1-42$ injection and CJC-1131 treatment groups $(A \beta 1-42$ injection: $F(1,38)=79.876$, $\mathrm{p}<0.001$; CJC-1131 treatment: $\mathrm{F}(1,38)=65.105, p<0.001 ; A \beta 1-42$ by CJC-1131 interaction: $F(1,38)=11.000, p<0.01$. Fig. $2 C, D)$. The slope of fEPSP in all groups had similar main effects and interactions (Aß1-42 injection: $F(1,38)=30.667, p<0.001$; CJC-1131 treatment: $F(1,38)=28.231, p<0.001 ; A \beta 1-42$ by CJC-1131 interaction: $F(1,38)=9.809, p<0.01$. Fig. $2 E, F)$. Tukey's post hoc test showed $A \beta 1-42$ injection induced a significant decrease in the amplitude and the slope of fEPSP $(p<0.001)$, while the decrease was effectively reversed by CJC-1131 treatment $(p<0.001)$.

\subsection{CJC-1131 restored the level of p-PKA in the hippocampus}

After finishing LTP recording, we examined the level of p-PKA in the hippocampus with the same animals. Fig. 3A displayed the Western blot bands of p-PKA and PKA in the hippocampus, with GAPDH as the loading control. As shown in Fig. 3B, two-way ANOVA showed that the main effects of the p-PKA level in A $\beta 1-42$ injection and CJC-1131 treatment were significant $(A \beta 1-42$ injection: $F_{(1,22)}=6.499, p<0.05 ; C J C-1131$ treatment: $F_{(1,22)}=4.602$, $p<0.05 ; A \beta 1-42$ by CJC-1131 interaction: $\left.\mathrm{F}_{(1,22)}=8.182 ; p<0.05\right)$. Tukey's post hoc test showed $A \beta 1-42$ injection induced a significant decrease in the level of p-PKA $(p<0.05)$, which was effectively prevented by CJC-1131 treatment $(p<0.05)$.

\section{Discussion}

The production and aggregation of $A \beta$ are involved in the learning and memory deficits in AD. Not only natural $A \beta$ such as $A \beta 1-40$ and $A \beta 1-42$, but also synthetic $A \beta$ fragments like $A \beta 25-35$ and even $A \beta 31-35$ have strong neurotoxicity [25]. In our previous study,
$A \beta 31-35$, similar to full length of $A \beta$, also induced apoptosis in cultured cortical neurons [26], formed transmembrane ion channels [27], suppressed potassium channels in neurons dissociated from the hippocampus $[28,29]$, and impaired in vivo hippocampal LTP and spatial memory in rats $[19,30]$.

It has been reported that $A \beta$ desensitizes insulin receptors in cultured hippocampal neurons as well as reducing expression of the receptors [31,32]. Interestingly, these effects of $A \beta$ can be reversed by activating GLP-1 receptor [33]. GLP-1, an insulinotropic endocrine hormone based on blood sugar concentration, can be applied to non-diabetic patients [34,35] and directly reduces endogenous $A \beta$ levels in the mouse brain [36]. GLP-1 also promotes neurite outgrowth $[37,38]$ and protects against oxidative injury and excitotoxic cell death [36]. In the present study, we used a new GLP-1 analogue CJC-1131, which was profoundly resistant to DPP IV degradation and has much longer half life [16]. We first confirmed in MWM test that treatment with CJC-1131 could effectively prevent against $A \beta 1-42$-induced escape latency increase in the hidden platform test, suggesting that CJC-1131 could ameliorate $A \beta 1-42$-induced impairment of spatial learning ability. Then, we found that $C J C-1131$ could effectively reversed $A \beta 1-42$ induced decrease of swimming time in the target quadrant in probe trial, which meant that spatial memory deficit induced by $A \beta 1-42$ could be avoided by CJC-1131. Moreover, the similar escape latency in the visible platform test and swimming speed between different groups excluded the possibility of visual or motor function deficits. We further investigated the effects of CJC-1131 on A 1 1-42-induced impairments of hippocampal synaptic plasticity. As expected, CJC1131 prevented against $A \beta 1-42$-induced LTP suppression, which probably explained that CJC-1131 could rescue spatial cognition impairments induced by $A \beta 1-42$.

These results are also supported by our previous study using other GLP-1 analogs. For instance, Val(8)-GLP-1 [39], liraglutide [5] and lixisenatide [19] also reversed the suppression of LTP and the impairments of spatial learning and memory in rats. Similarly, During [38] found that the learning deficit appeared in GLP-1R-deficient mice was improved after hippocampal GLP-1R gene transfer. In addition, over expressing GLP-1R in the hippocampus also improved learning and memory of rats; application of N-AcGIP, an enzyme-resistant analogue of glucose-dependent insulinotropic polypeptide (GIP), facilitated hippocampal LTP and reversed the impairment of LTP induced by $A \beta$ [40].

One of the possible mechanisms by which CJC-1131 prevents $A \beta 1-42$-induced deficits in spatial cognition and synaptic plasticity could be the activation of the CAMP/PKA pathway. It is reported that $\mathrm{A} \beta$ can induce cytoplasmic $\mathrm{Ca}^{2+}$ overload [41], which results in the inhibition of AC [42], and thereby turns off a cascade reaction such as activation of cAMP and PKA. For instance, Velmurugan $\mathrm{K}$ found that $A \beta$ inhibited the activity of PKA in mice cortical neurons [43]; 
Wang reported that $A \beta$-induced inhibition of LTP was prevented by forskolin, an activator of AC [44]; Vitolo found that A $\beta$-induced impairment of LTP was mediated through the inactivation of PKA [45]. Recently, we also found that the injection of GLP-1 agonist liraglutide could effectively increase the level of cAMP in rats brain [5]. So, we further examined the effects of GLP-1 agonist on the activity of PKA in the present study. Our results showed that $A \beta 1-42$ effectively decreased the level of p-PKA and this down-regulation could be effectively reversed by CJC-1131. It is well known that the up-regulated cAMP/PKA activity could promote gene transcription in the nucleus and finally might enhance learning and memory behavior. Therefore, we propose that the activation of CAMP/PKA pathway might be important for the neuroprotective function of GLP-1 analogs, while CJC-1131 itself could be a novel and promising candidate to protect learning and memory in neurodegenerative diseases such as AD.

\section{Acknowledgements}

This project was supported by National Science Foundation of China (31471080, 31271201), Graduate Students Outstanding Innovation Project Foundation of Shanxi Province (20143061) and National Undergraduate Innovation Program of China (201310114007, 201410114007).

\section{References}

[1] C. Zhang, Natural compounds that modulate BACE1-processing of amyloid-beta precursor protein in Alzheimer's disease, Discov. Med. 14 (76) (2012) 189-197.

[2] L. Frings, T.S. Spehl, W.A. Weber, M. Hull, P.T. Meyer, Amyloid-beta load predicts medial temporal lobe dysfunction in alzheimer dementia, J. Nucl. Med. 54 (November (11)) (2013) 1909-1914.

[3] S.S. Tang, H. Hong, L. Chen, Z.L. Mei, M.J. Ji, G.Q. Xiang, N. Li, H. Ji, Involvement of cysteinyl leukotriene receptor 1 in Abeta1-42-induced neurotoxicity in vitro and in vivo, Neurobiol. Aging 35 (3) (2014) 590-599.

[4] X.H. Wang, L. Li, C. Holscher, Y.F. Pan, X.R. Chen, J.S. Qi, Val8-glucagon-like peptide-1 protects against Abeta1-40-induced impairment of hippocampal late-phase long-term potentiation and spatial learning in rats, Neuroscience 170 (4) (2010) 1239-1248.

[5] W.-N. Han, C. Hölscher, L. Yuan, W. Yang, X.-H. Wang, M.-N. Wu, J.-S. Qi, Liraglutide protects against amyloid- $\beta$ protein-induced impairment of spatial learning and memory in rats, Neurobiol. Aging 34 (2) (2013) 576-588.

[6] Z.J. Wang, W.N. Han, G.Z. Yang, L. Yuan, X.J. Liu, Q.S. Li, J.S. Qi, The neuroprotection of Rattin against amyloid beta peptide in spatial memory and synaptic plasticity of rats, Hippocampus 24 (1) (2014) 44-53.

[7] P. Toro, P. Schonknecht, J. Schroder, Type II diabetes in mild cognitive impairment and Alzheimer's disease: results from a prospective population-based study in Germany, J. Alzheimers Dis. 16 (4) (2009) 687-691.

[8] M.W. Strachan, R.M. Reynolds, R.E. Marioni, J.F. Price, Cognitive function, dementia and type 2 diabetes mellitus in the elderly, Nat. Rev. Endocrinol. 7 (2) (2011) 108-114

[9] M. Watve, A. Bodas, M. Diwekar, Altered autonomic inputs as a cause of pancreatic beta-cell amyloid, Med. Hypotheses 82 (1) (2014) 49-53.

[10] J. Miklossy, H. Qing, A. Radenovic, A. Kis, B. Vileno, F. Laszlo, L. Miller, R.N. Martins, G. Waeber, V. Mooser, F. Bosman, K. Khalili, N. Darbinian, P.L. McGeer, Beta amyloid and hyperphosphorylated tau deposits in the pancreas in type 2 diabetes, Neurobiol. Aging 31 (9) (2010) 1503-1515.

[11] Y. Chen, Z. Liang, J. Blanchard, C.L. Dai, S. Sun, M.H. Lee, I. Grundke-Iqbal, K Iqbal, F. Liu, C.X. Gong, A non-transgenic mouse model (icv-STZ mouse) of Alzheimer's disease: similarities to and differences from the transgenic model (3xTg-AD mouse), Mol. Neurobiol. 47 (2) (2013) 711-725.

[12] M.W. Schwartz, A. Sipols, S.E. Kahn, D.F. Lattemann, G.J. Taborsky Jr., R.N. Bergman, S.C. Woods, D. Porte Jr., Kinetics and specificity of insulin uptake from plasma into cerebrospinal fluid, Am. J. Physiol. 259 (3 Pt 1) (1990) E378-E383

[13] Z. Kroner, The relationship between alzheimer's disease and diabetes: type 3 diabetes, Altern. Med. Rev. 14 (4) (2009) 373-379.

[14] C.W. Chia, J.M. Egan, Biology and therapeutic potential of GLP-1 in the treatment of diabetes, Drug Discovery Today: Dis. Mech. 2 (3) (2005) 295-301.

[15] R. Leger, K. Thibaudeau, M. Robitaille, O. Quraishi, P. van Wyk, N. Bousquet-Gagnon, J. Carette, J.P. Castaigne, D.P. Bridon, Identification of CJC-1131-albumin bioconjugate as a stable and bioactive GLP-1(7-36) analog, Bioorg. Med. Chem. Lett. 14 (17) (2004) 4395-4398.

[16] B. Lawrence, J., Dreyfus, S., Wen, P. Guivarc'h, D., Drucker, J., Castaigne, CJC-1131, a long acting GLP-1 derivative, exhibits an extended pharmacokinetic profile in healthy human volunteers, Diabetes, AMER
DIABETES ASSOC $1701 \mathrm{~N}$ BEAUREGARD ST, ALEXANDRIA, VA 22311-1717 USA, (2003) A125.

[17] R.G. Tiessen, J.P. Castaigne, J.F. Dreyfus, M. Nemansky, H.H. Kruizinga, A.A. van Vliet, Pharmacokinetics and tolerability of a novel long-acting glucagon-like peptide-1 analog, CJC-1131, in healthy and diabetic subjects, Int. J. Clin. Pharmacol. Ther. 46 (9) (2008) 443-452.

[18] L. Blonde, Clinical trial results of GLP-1-related agents: the early evidence, Adv Stud. Med. 5 (10) (2005) S1074-S1078

[19] H.Y. Cai, C. Holscher, X.H. Yue, S.X. Zhang, X.H. Wang, F. Qiao, W. Yang, J.S. Qi, Lixisenatide rescues spatial memory and synaptic plasticity from amyloid beta protein-induced impairments in rats, Neuroscience 277 (2014) 6-13.

[20] W.N. Han, C. Holscher, L. Yuan, W. Yang, X.H. Wang, M.N. Wu, J.S. Qi, Liraglutide protects against amyloid-beta protein-induced impairment of spatial learning and memory in rats, Neurobiol. Aging 34 (2) (2013) 576-588.

[21] J.K. Ryu, J.G. McLarnon, Minocycline or iNOS inhibition block 3-nitrotyrosine increases and blood-brain barrier leakiness in amyloid beta-peptide-injected rat hippocampus, Exp. Neurol. 198 (2) (2006) 552-557.

[22] R. Morris, Developments of a water-maze procedure for studying spatial learning in the rat, J. Neurosci. Methods 11 (1) (1984) 47-60.

[23] A.V. Terry, Jr., Spatial Navigation (Water Maze) Tasks Methods of Behavior Analysis in Neuroscience, 2009 (Chapter 13)

[24] T.V. Bliss, G.L. Collingridge, A synaptic model of memory: long-term potentiation in the hippocampus, Nature 361 (6407) (1993) 31-39.

[25] L. Yuan, X.J. Liu, W.N. Han, Q.S. Li, Z.J. Wang, M.N. Wu, W. Yang, J.S. Qi, [Gly14]-Humanin protects against amyloid beta peptide-induced impairment of spatial learning and memory in rats, Neurosci Bull Aug 32 (4) (2016) 374-382.

[26] X.Z. Yan, J.T. Qiao, Y. Dou, Z.D. Qiao, Beta-amyloid peptide fragment 31-35 induces apoptosis in cultured cortical neurons, Neuroscience 92 (1) (1999) $177-184$.

[27] J.S. Qi, J.T. Qiao, Amyloid beta-protein fragment 31-35 forms ion channels in membrane patches excised from rat hippocampal neurons, Neuroscience 105 (4) (2001) 845-852.

[28] J.S. Qi, J.T. Qiao, Suppression of large conductance Ca2+-activated K+ channels by amyloid beta-protein fragment 31-35 in membrane patches excised from hippocampal neurons, Sheng Li Xue Bao 53 (3) (2001) 198-204.

[29] J.S. Qi, L. Ye, J.T. Qiao, Amyloid beta-protein fragment 31-35 suppresses delayed rectifying potassium channels in membrane patches excised from hippocampal neurons in rats, Synapse 51 (3) (2004) 165-172.

[30] S.F. Li, M.N. Wu, X.H. Wang, L. Yuan, D. Yang, J.S. Qi, Requirement of alpha7 nicotinic acetylcholine receptors for amyloid beta protein-induced depression of hippocampal long-term potentiation in CA1 region of rats in vivo, Synapse 65 (11) (2011) 1136-1143.

[31] L. Xie, E. Helmerhorst, K. Taddei, B. Plewright, W. Van Bronswijk, R. Martins, Alzheimer's beta-amyloid peptides compete for insulin binding to the insulin receptor, J. Neurosci. 22 (10) (2002) (RC221)

[32] W.Q. Zhao, F.G. De Felice, S. Fernandez, H. Chen, M.P. Lambert, M.J. Quon, G.A. Krafft, W.L. Klein, Amyloid beta oligomers induce impairment of neuronal insulin receptors, FASEB J. 2 (1) (2008) 246-260.

[33] H. Gao, X. Wang, Z. Zhang, Y. Yang, J. Yang, X. Li, G. Ning, GLP-1 amplifies insulin signaling by up-regulation of IRbeta, IRS-1 and Glut4 in 3T3-L1 adipocytes, Endocrine 32 (1) (2007) 90-95.

[34] D.J. Drucker, Enhancing incretin action for the treatment of type 2 diabetes, Diabetes Care 26 (10) (2003) 2929-2940.

[35] B. Gallwitz, Therapies for the treatment of type 2 diabetes mellitus based on incretin action, Minerva Endocrinol. 31 (2) (2006) 133-147.

[36] T. Perry, D.K. Lahiri, K. Sambamurti, D. Chen, M.P. Mattson, J.M. Egan, N.H. Greig, Glucagon-like peptide-1 decreases endogenous amyloid-beta peptide (Abeta) levels and protects hippocampal neurons from death induced by Abeta and iron, J. Neurosci. Res. 2 (5) (2003) 603-612.

[37] T. Perry, D.K. Lahiri, D. Chen, J. Zhou, K.T. Shaw, J.M. Egan, N.H. Greig, A novel neurotrophic property of glucagon-like peptide 1: a promoter of nerve growth factor-mediated differentiation in PC12 cells, J. Pharmacol. Exp. Ther. 300 (3) (2002) 958-966.

[38] M.J. During, L. Cao, D.S. Zuzga, J.S. Francis, H.L. Fitzsimons, X. Jiao, R.J. Bland, M. Klugmann, W.A. Banks, D.J. Drucker, C.N. Haile, Glucagon-like peptide-1 receptor is involved in learning and neuroprotection, Nat. Med. 9 (9) (2003) 1173-1179.

[39] X.H. Wang, L. Li, C. Hölscher, Y.F. Pan, X.R. Chen, J.S. Qi, Val8-glucagon-like peptide- 1 protects against $A \beta 1-40$-induced impairment of hippocampal late-phase long-term potentiation and spatial learning in rats, Neuroscience 170 (4) (2010) 1239-1248.

[40] V.A. Gault, C. Holscher, Protease-resistant glucose-dependent insulinotropic polypeptide agonists facilitate hippocampal LTP and reverse the impairment of LTP induced by beta-amyloid, J. Neurophysiol. 99 (4) (2008) 1590-1595.

[41] X.H. Wang, W. Yang, C. Holscher, Z.J. Wang, H.Y. Cai, Q.S. Li, J.S. Qi, Val(8)-GLP-1 remodels synaptic activity and intracellular calcium homeostasis impaired by amyloid beta peptide in rats, J. Neurosci. Res. 91 (4) (2013) 568-577

[42] S.T. Wong, J. Athos, X.A. Figueroa, V.V. Pineda, M.L. Schaefer, C.C. Chavkin, L.J. Muglia, D.R. Storm, Calcium-stimulated adenylyl cyclase activity is critical for hippocampus-dependent long-term memory and late phase LTP, Neuron 23 (4) (1999) 787-798

[43] H. Du, L. Guo, X. Wu, A.A. Sosunov, G.M. McKhann, J.X. Chen, S.S. Yan, Cyclophilin D deficiency rescues Abeta-impaired PKA/CREB signaling and 
alleviates synaptic degeneration, Biochim. Biophys. Acta 1842 (12 Pt A) (2014) 2517-2527.

[44] Q.W. Wang, M.J. Rowan, R. Anwyl, Inhibition of LTP by beta-amyloid is prevented by activation of beta2 adrenoceptors and stimulation of the cAMP/PKA signalling pathway, Neurobiol. Aging 30 (10) (2009) 1608-1613.
[45] O.V. Vitolo, A. Sant'Angelo, V. Costanzo, F. Battaglia, O. Arancio, M. Shelanski, Amyloid beta - peptide inhibition of the PKA/CREB pathway and long-term potentiation: reversibility by drugs that enhance cAMP signaling, Proc. Natl. Acad. Sci. U. S. A. 99 (20) (2002) 13217-13221. 